\title{
Comparação de técnicas de ovariosalpingohisterectomia em cadelas
}

\author{
Comparison among ovariohysterectomy techniques in bitches \\ Ana Maria Quessada', Antonio Augusto Rodrigues de Sousa ${ }^{2}$, Amiltom Paulo Raposo Costa', \\ Adriana Abreu Soares de Sousa ${ }^{2}$ \& Raimundo Rômulo Costa Rocha ${ }^{3}$
}

\begin{abstract}
RESUMO
A ovariosalpingohisterectomia $(\mathrm{OSH})$ em cadelas é a cirurgia mais frequentemente realizada na prática clínica veterinária, principalmente com a finalidade de evitar a reprodução. Em estudo comparativo de duas técnicas, em dez cadelas, foram realizadas OSH através de duas incisões paramedianas (direita e esquerda), para ligadura dos ovários. Foi realizada ainda uma incisão mediana pélvica para ligadura do coto uterino e retirada dos órgãos. Esta primeira técnica foi denominada experimental. Em outros 10 animais, houve uma única incisão pré-retroumbilical para a retirada dos ovários e útero (após ligaduras desses órgãos), em procedimento nomeado de técnica tradicional. Os animais foram avaliados clinicamente e foi também mensurado o cortisol plasmático. As avaliações foram feitas no pré-operatório e se estenderam no trans e pós-operatório. A técnica experimental provoca maior estresse, mas pode ser indicada em mutirões de castração, por não apresentar complicações pós-operatórias imediatas.
\end{abstract}

Descritores: canino, castração, cirurgia, ovário, útero.

\begin{abstract}
The ovariohisterectomy $(\mathrm{OH})$ in bitches is the most commonly performed surgery in veterinary clinical practice, mainly in order to avoid reproduction. In a comparative study of two techniques, were performed $\mathrm{OH}$ in ten bitches through two paramedian incisions (right and left) for ligation of the ovaries. It also held a median pelvic incision for ligation of uterine stump. After ligation, ovaries and uterus were removed. This first technique is called experimental. In another 10 animals had a single preretroumbilical incision for ligation of the ovaries and uterus. After ligation, these organs were removed. This last procedure was named traditional technique. The animals were evaluated clinically. The plasmatic cortisol was also measured. All these data were collected preoperatively, and these measurements extended during surgery and post-operatively. The experimental technique causes more stress, but may be indicated for massal castration due to not presenting immediate postoperative complications.
\end{abstract}

Keywords: canine, castration, ovary, surgery, uterus.

Trabalho originado da Dissertação do segundo autor. Curso de Pós-graduação em Ciência Animal (PPGCA), Universidade Federal do Piauí (UFPI), Campus Universitário Ministro Petrônio Portella, Bairro Ipitinga, CEP 64049-550 Teresina, PI, Brasil. 'Departamento de Clínica e Cirurgia Veterinária, Curso de Medicina Veterinária, Centro de Ciências Agrárias (CCA)-UFPI. ${ }^{2}$ Universidade Estadual do Maranhão (UEMA), São Luís, MA, Brasil. ${ }^{3}$ Secretaria de Agricultura do Estado do Maranhão, São Luís, MA. CORRESPONDÊNCIA: A.M. Quessada [quessadavet@gmail.com ; Fax: + 55 (86) 3215 5743]. 


\section{INTRODUÇÃO}

A ovariosalpingohisterectomia $(\mathrm{OSH})$ em cadelas é a cirurgia eletiva mais realizada em clínicas e hospitais veterinários [6]. As principais indicações de OSH são controle da natalidade [2], partos distócicos, prevenção de tumores mamários, estros prolongados, tratamento de enfermidades ovarianas, uterinas e vaginais $[2,5,6]$.

São várias as técnicas cirúrgicas utilizadas para esterilização em cadelas, incluindo OSH por incisão mediana pré-retroumbilical e OSH laparoscópica [1,7].

Técnicas minimamente invasivas são ideais para mutirões de castração, já que permitem que o animal retorne para casa logo que se recupere da anestesia. Além disso, evita muito tempo de hospitalização, diminuindo o custo do procedimento e o estresse do paciente [8].

A principal desvantagem das cirurgias minimamente invasivas é o tempo de execução, que pode ser reduzido pelo treinamento da equipe cirúrgica, para minimizar ou suprimir as dificuldades técnicas [7].

Esta pesquisa tem por objetivo comparar duas técnicas de OSH em cadelas para emprego em mutirões de castração. $\mathrm{O}$ experimento foi aprovado pelo Comitê de Ética em Pesquisa da instituição de origem.

\section{MATERIAS E MÉTODOS}

Foram utilizadas vinte cadelas, sem raça definida (SRD), divididas em dois grupos iguais (GI e GII), com peso médio de $15 \mathrm{~kg}$, com diferentes idades. Foram selecionadas para o experimento apenas as cadelas que apresentaram resultados normais no exame clínico, hemograma completo e sorologia para leishmaniose visceral canina. Em todos os animais, foi

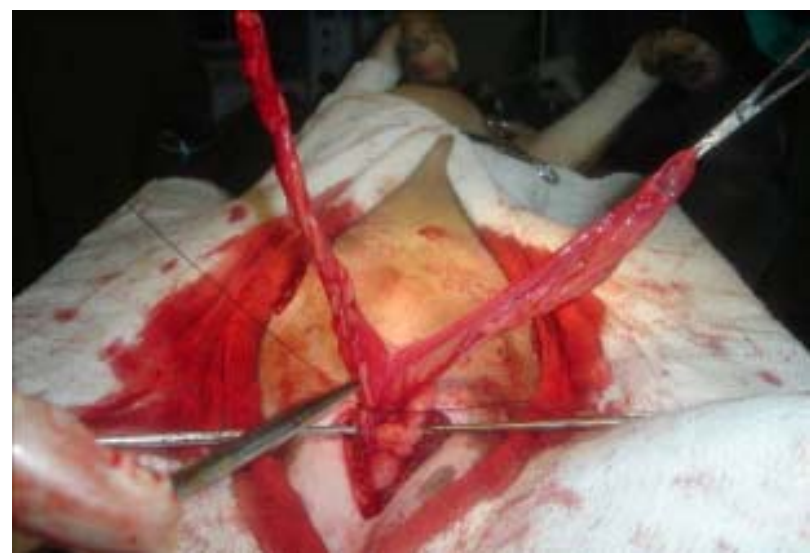

Figura 1. Transfixação uterina, ligadura individual das artérias uterinas e retirada do útero por incisão pélvica mediana em cadela. feita profilaxia antibiótica com penicilina benzatina ${ }^{1}$ (40.000/ui/kg, por via intramuscular) e analgesia preemptiva com flunixin meglumine ${ }^{2}(1,1 \mathrm{mg} / \mathrm{kg}$ por via intramuscular).

$\mathrm{Na}$ indução anestésica, foi utilizada, para os animais de ambos os grupos, quetamina ${ }^{3}(4 \mathrm{mg} / \mathrm{kg})$ associada ao diazepam $(0,5 \mathrm{mg} / \mathrm{kg})$, por via intravenosa. A anestesia foi mantida com halotano ${ }^{5}$, em circuito semifechado. Foi administrada solução de ringer lactato $^{6}$, na dose de $10 \mathrm{ml} / \mathrm{kg} /$ hora, durante todo o transoperatório.

Antes da realização de quaisquer procedimentos, foram avaliadas, em todos os animais, a frequência respiratória (FR) e a frequência cardíaca (FC). Este momento de avaliação pré-cirúrgica foi denominado $\mathrm{M}_{0}$. Estes parâmetros foram também mensurados no trans e pós-operatórios, em momentos denominados $\mathrm{M}_{1}$ (imediatamente após indução anestésica no início da cirurgia); $\mathrm{M}_{2}$ (no momento de pinçamento dos ovários); $\mathrm{M}_{3}$ (final da cirurgia); $M_{4}$ (retorno anestésico); $M_{5}$ (deambulação); $M_{6}$ (micção espontânea); $M_{7}$ (alimentação espontânea); $M_{8}$ (12 horas após $\mathbf{M}_{1}$ ); $\mathbf{M}_{9}$ (vinte e quatro horas após $\mathbf{M}_{1}$ ) e $M_{10}$ (quarenta e oito horas após $M_{1}$ ). Mensurou-se o cortisol plasmático (CP) em todos os animais, desde $\mathrm{M}_{1}$ até $\mathrm{M}_{10}$. A concentração do cortisol foi determinada pela técnica de radioimunoensaio.

Nos animais do GI, foi realizada OSH por uma técnica cirúrgica minimamente invasiva, constando de três incisões: duas paramedianas (direita e esquerda) e uma mediana pélvica. As incisões paramedianas compreenderam de 2 a $3 \mathrm{~cm}$, em sentido longitudinal seguindo a disposição das fibras musculares, tendo como referência a terceira e quarta mamas. Após incisão da pele, subcutâneo, músculos (oblíquo externo, oblíquo interno, transverso) e peritônio, os ovários foram exteriorizados

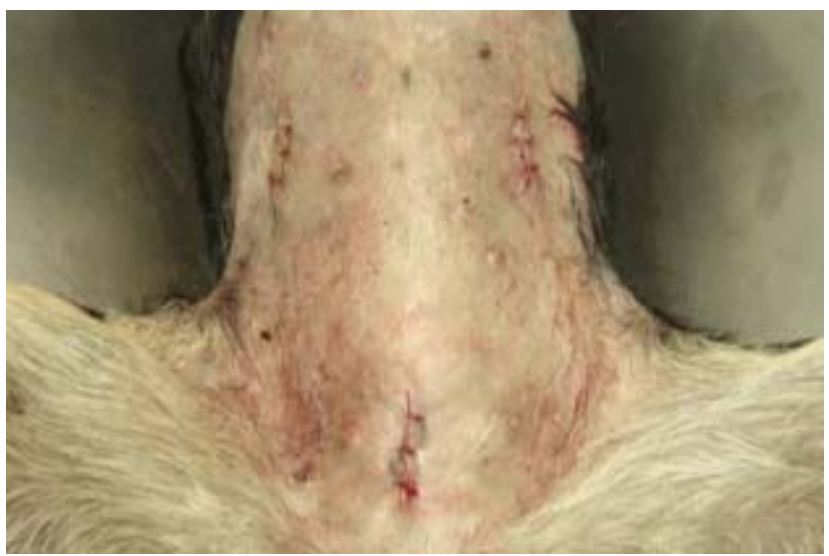

Figura 2. Suturas cutâneas após realização de ovariosalpingohisterectomia em cadela, através de três incisões (duas paramedianas e uma pélvica). 
com a utilização do gancho de Snook. Foi feito uma fenestra no ligamento largo para passagem do fio de sutura. As ligaduras dos pedículos ovarianos e transfixação foram feitas com fio de náilon 2-0. As extremidades dos cornos uterinos foram mantidas com fios longos e ancoradas com pinças hemostáticas. Em seguida, foi realizada incisão pélvica na linha média, próxima ao púbis, exteriorizando-se o útero e os ovários, por tração em direção à incisão. Foi feita ligadura bilateral das artérias uterinas e o útero foi transfixado e ligado. Os órgãos foram retirados pela incisão pélvica (Figura 1). A cavidade foi fechada em dois planos de sutura. $\mathrm{O}$ primeiro abrangeu peritônio, fáscia e músculos (oblíquo externo, oblíquo interno, transverso) e o segundo, subcutâneo e pele. Nos dois planos, utilizou-se sutura em pontos simples separados, com fio de náilon 2-0 (Figura 2).

Nas cadelas do GII foi realizada OSH, por incisão pré-retroumbilical na linha média em técnica tradicional [5]. Os ovários foram localizados e exteriorizados. Os pedículos ovarianos foram ligados. A artéria uterina foi ligada bilateralmente. $\mathrm{O}$ útero foi ligado por transfixação e os órgãos foram retirados. Todas as ligaduras foram feitas com fio de náilon 2-0. A cavidade foi fechada em três planos. O primeiro abrangeu peritônio, fáscia e músculos, utilizando-se sutura em pontos simples separados. O segundo abrangeu o tecido subcutâneo e foi realizado com pontos tipo cushing. A pele foi fechada com pontos separados simples. Todas as suturas foram feitas com fio de náilon 2-0.

Após o procedimento cirúrgico, todas as cadelas foram internadas e acompanhadas clinicamente durante 48 horas, período em que foram observadas as possíveis complicações. Durante o internamento, foi administrado tramadol ${ }^{7}$ na dose de $2 \mathrm{mg} / \mathrm{kg}$ de $8 / 8$ horas. Dois dias após a cirurgia, os animais tiveram alta. Uma semana após a cirurgia, foram retirados os pontos cutâneos, momento no qual as cadelas foram novamente examinadas e anotadas quaisquer alterações.

A avaliação estatística foi realizada por meio de análise de variância, seguida pelo teste Student Newman Keuls para comparação das médias dos diferentes tempos de observação dentro do mesmo grupo e entre grupos, com nível de significância estipulado em $5 \%(\mathrm{P}<0.05)$.

\section{RESULTADOS}

Na média geral, a FR foi menor nas cadelas da técnica experimental do que nas cadelas da técnica tradicional (Figura 3) $(\mathrm{p}<0,05)$, mostrando aparentemente um procedimento mais estressante na técnica tradicional. Na evolução da FR ao longo dos procedimentos, observou-se que houve redução significativa da FR, em ambos os grupos, entre o pré-operatório e o início da cirurgia, sendo que nos animais da técnica experimental retornou ao valor inicial no final da cirurgia, enquanto nos animais da técnica tradicional retornou durante o pinçamento dos ovários (transoperatório). No pós-operatório, quando as cadelas urinaram e se alimentaram espontaneamente, houve nova redução da FR nos animais da técnica experimental, voltando aos valores iniciais (Figura 3).

A FC não foi diferente entre os grupos na média geral (Figura 4). Entretanto, houve diferença significativa no início da cirurgia, sendo maior nos animais da técnica tradicional $(\mathrm{p}<0,05)$. Quanto à evolução, ao

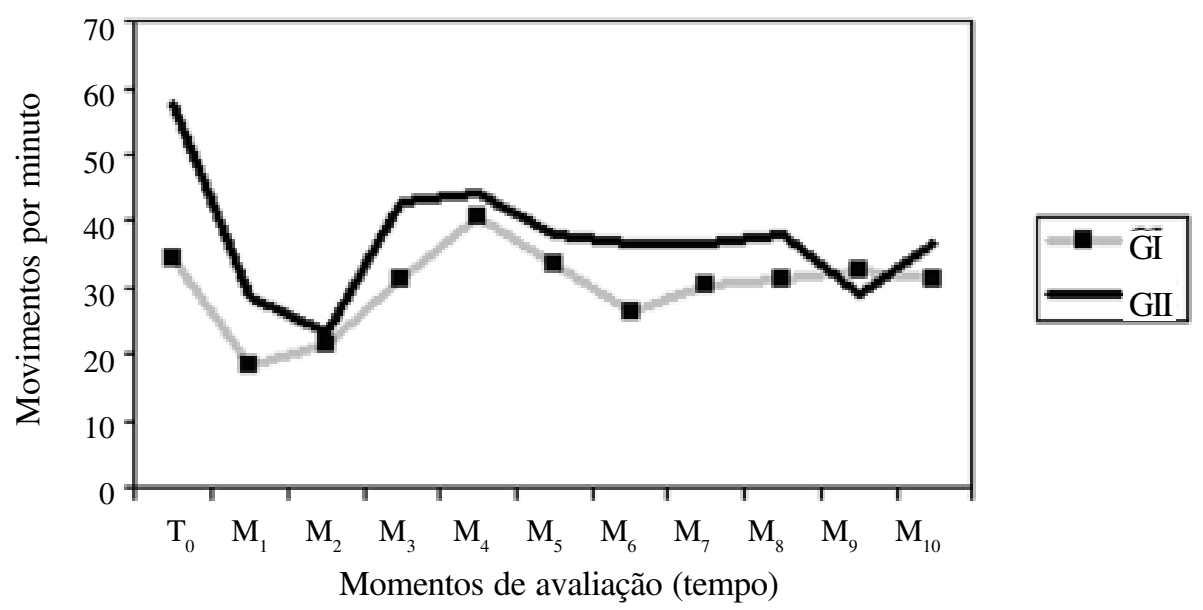

GI: Técnica de três incisões (duas paramedianas e uma pélvica). GII: Técnica tradicional (incisão mediana pré-retroumbilical). $\mathrm{T}_{0}$ : pré-operatório; $\mathrm{T}_{1}$ : início da cirurgia; $\mathrm{T}_{10}: 48$ horas após $\mathrm{T}_{1}$ ).

Figura 3. Médias de frequência respiratória de cadelas submetidas a duas técnicas de ovariosalpingohisterectomia. 
Quessada A.M., Sousa A.A.R., Costa A.P.R., Sousa A.A.S.\& Rocha R.R.C. 2009. Comparação de técnicas de ovariosalpingohis-

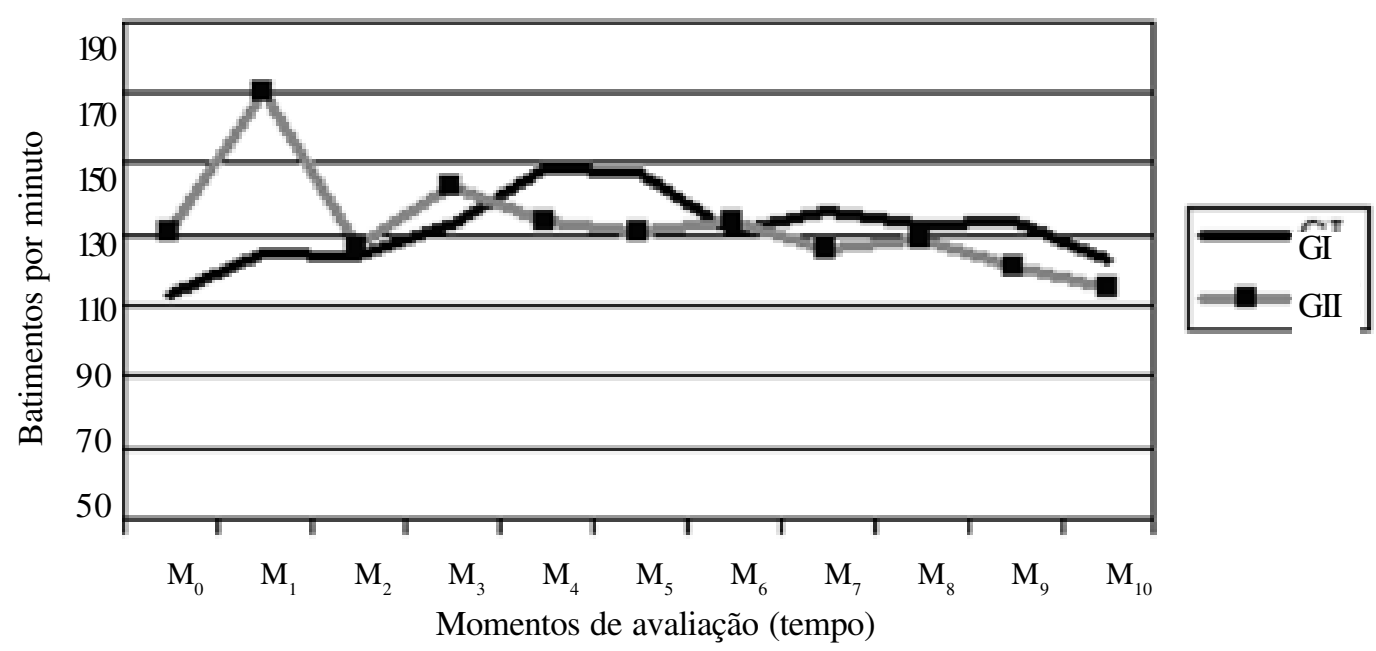

GI: Técnica de três incisões (duas paramedianas e uma pélvica). GII: Técnica tradicional (incisão mediana pré-retroumbilical). $\mathrm{M}_{0}$ : pré-operatório; $\mathrm{M}_{1}$ : início da cirurgia; $\mathrm{M}_{10}: 48$ horas após $\mathrm{M}_{1}$ ).

Figura 4. Médias de frequência cardíaca de cadelas submetidas a duas técnicas de ovariosalpingohisterectomia.

longo do tempo e com os procedimentos, as cadelas da técnica tradicional apresentaram aumento da FC no início da cirurgia, retornando ao valor inicial logo depois (no momento do pinçamento dos ovários) (Figura 4). Nas cadelas da técnica experimental, também houve aumento significativo no início da cirurgia. A FC se manteve significativamente mais elevada do que a inicial até as 48 horas de pós-operatório (PO).

O cortisol plasmático não teve diferença estatisticamente significativa entre os grupos, na média geral. Quanto à evolução ao longo dos procedimentos cirúrgicos e do pós-operatório, ambos os grupos tiveram aumento significativo, logo após a indução $(\mathrm{p}<0,05)$ (Figura 5). Esses valores permaneceram suprafisiológicos até as $24 \mathrm{~h}$ do pós-operatório nas cadelas da técnica experimental e até as $12 \mathrm{~h}$ nas cadelas da técnica tradicional, mostrando uma maior duração do estresse nos animais da técnica experimental.

Os tempos cirúrgicos foram estatisticamente diferentes $(\mathrm{P}<0,05)$, sendo que, no procedimento cirúrgico realizado na técnica experimental, a duração foi maior do que na técnica tradicional.

Nas cadelas da técnica experimental, não foram observadas complicações pós-operatórias imediatas como seroma, inflamação, eventração e evisceração. $\mathrm{Na}$ técnica tradicional, quatro animais apresentaram seroma após o ato cirúrgico, nas primeiras 24 horas.

\section{DISCUSSÃO}

O valor inicial da FR nas cadelas da técnica tradicional foi suprafisiológico, aparentemente por estresse devido aos procedimentos de contenção, considerando-se que os valores normais para espécie canina, em repouso, podem variar de 20 a 34 movimentos respiratórios por minuto [9]. O aumento da FR está ligado ao estresse [10].

Vale salientar que a FR permaneceu, a maior parte do tempo, dentro dos níveis fisiológicos [9] nas cadelas dos dois grupos (Figura 3). Apenas nas cadelas da técnica experimental, no início da cirurgia, ocorreu uma diminuição significativa da FR (Figura 3). No entanto, isso não acarretou complicações clínicas que pudessem ser atribuídas ao evento.

A diminuição da FR no início da cirurgia pode estar relacionada à indução anestésica [11]. Além disso, são esperadas variações sobre este parâmetro em anestesias de cães [4].

$\mathrm{O}$ aumento da FC no início da cirurgia, nos animais dos dois grupos, provavelmente, deve-se ao estresse de contenção, aos procedimentos anestésicos e ao trauma cirúrgico inicial $[3,11]$. O aumento da FC no pós-operatório provavelmente foi relacionado ao estresse de internação, já que foi utilizado analgésico para controle da dor.

A elevação dos níveis plasmáticos de cortisol acima dos valores de referência em cães, que variam de 0,5-6,0 $\mu \mathrm{g} / \mathrm{dl}$ [3], reflete o estresse e a dor inerentes ao procedimento cirúrgico e ao pós-operatório [7]. Nas cadelas da técnica experimental, a maior duração do estresse se deveu, possivelmente, à maior manipulação dos órgãos [7].

Embora seja considerada uma desvantagem, a duração maior do procedimento realizado nas cadelas da técnica experimental já era esperada, pois técnicas 


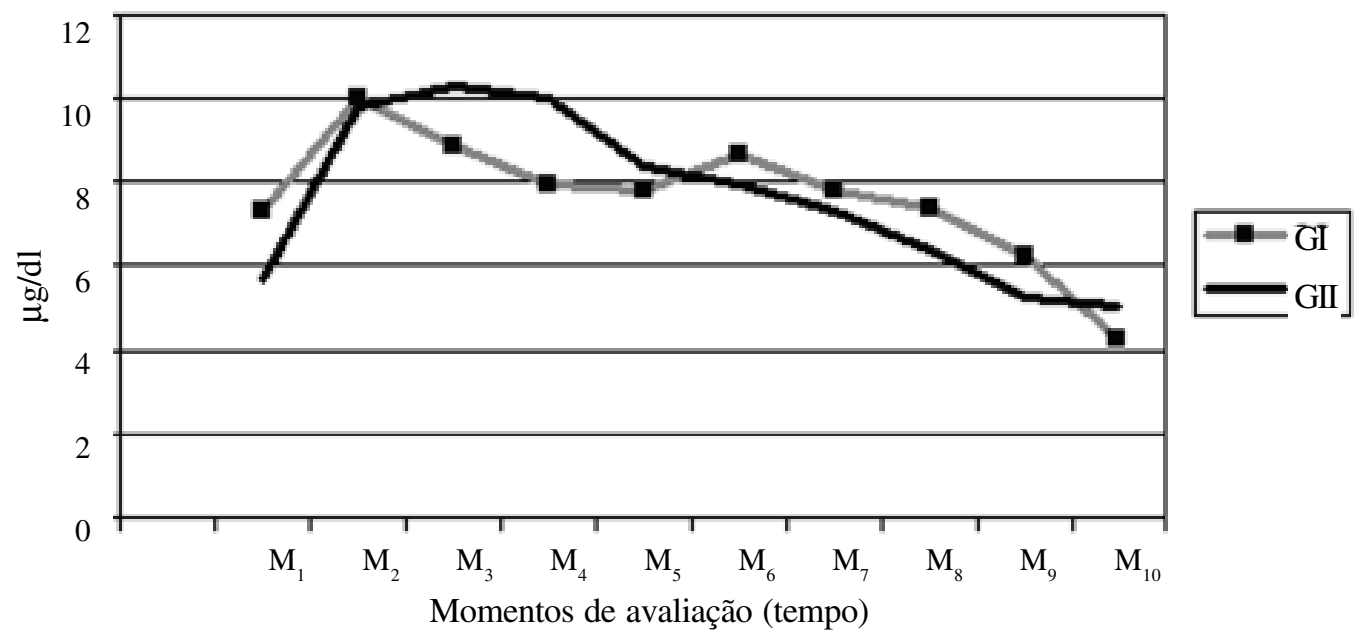

GI: Técnica de três incisões (duas paramedianas e uma pélvica). GII: Técnica tradicional (incisão mediana pré-retroumbilical). $M_{0}$ : pré-operatório; $M_{1:}$ início da cirurgia; $M_{10}: 48$ horas após $M_{1}$ )

Figura 5. Médias de cortisol plasmático em cadelas submetidas a duas técnicas de ovariosalpingohisterectomia.

minimamente invasivas demandam maior tempo de realização. Esta desvantagem pode diminuir com a experiência do cirurgião [7].

A ausência de complicações pós-operatórias imediatas é sempre observada em cirurgias minimamente invasivas [1].

\section{CONCLUSÕES}

A técnica de OSH considerada minimamente invasiva demonstrou ser segura e eficiente, podendo vir a ser empregada nos mutirões de castração, desde que haja treinamento da equipe para redução do tempo cirúrgico. O emprego em mutirões de castração é conveniente por não ocorrerem complicações pós-ope- ratórias imediatas, o que permite o retorno do animal para casa assim que se recuperar da anestesia. Além disso, sugere-se que a técnica experimental pode ser empregada com sucesso em cadelas obesas, nas quais é difícil acessar os ovários por via mediana.

\section{NOTAS INFORMATIVAS}

${ }^{1}$ Pen ve, Eurofarma, São Paulo-SP, Brasil.

${ }^{2}$ Flunamine, Bayer, São Paulo-SP, Brasil.

${ }^{3}$ Diazepam, Germed EMS, Hortolândia-SP, Brasil.

${ }^{4}$ Fluothane, Astrazeneca, Cotia-SP, Brasil.

${ }^{5}$ Francotar, Virbac São Paulo-SP, Brasil.

${ }^{6}$ Ringer com lactato, Equiplex, Aparecida de Goiânia-GO, Brasil.

${ }^{7}$ Cloridrato de tramadol, EMS, Hortolândia-SP, Brasil.

\section{REFERÊNCIAS}

1 Burrow R., Batchelor D. \& Cripps P. 2005. Complications observed during and after ovariohysterectomy of 142 bitches at a veterinary teaching hospital. Veterinary Record. 157: 829-833.

2 Concannon W.P. 1997. Endocrinologia reprodutiva, contracepção e terminação da gestação em cães. In: Ettinger J.S. \& Feldman C.E. (Eds). Tratado de medicina interna veterinária. 4. ed. São Paulo: Manole, pp.2242-2247.

3 Feldman D.F. \& Nelson R.W. 1996. The adrenal gland. In: Canine and feline endocrinology and reproduction. 2 nd edn. Philadelphia: Saunders, pp.187-265.

4 Hatschbach E., Massone F., José Von G.G.S. \& Lílian S.B. 2006. Parametria da associação do midazolam ou diazepam em cães pré-tratados pela atropina e tratados pela demedetomidina e quetamina. Ciência Rural. 36: 536-543.

5 Hedlund S.C. 2002. Cirurgia dos sistemas reprodutivo e genital. In: Fossum W.T. (Ed). Cirurgia de pequenos animais. São Paulo: Roca, pp.571-637.

6 Howe M.L. 2006. Surgical methods of contraception and sterilization. Theriogenology. 66: 500-509,

7 Malm C., Savassi-Rocha R.P., Gheller A.V., Oliveira P.H., Lamounie, R.A. \& Foltynek V. 2005. Ovariohisterectomia: estudo experimental comparativo entre as abordagens laparoscópica e aberta na espécie canina - III. Estresse pela análise do cortisol plasmático. Arquivo Brasileiro de Medicina Veterinária e Zootecnia. 57: 584-590.

8 Minami S., Okamoto Y., Eugchi H. \& Kato K. 1997. Sucessful laparoscopy assisted ovariohysterectomy in two dogs with pyometra. Journal Veterinary Medical Science. 159: 845-847. 
9 Reece W.O. 1996. Respiração nos mamíferos. In: Swenson M.J. \& Reece W.O. (Eds). Dukes/Fisiologia dos animais domésticos. 11.ed. Rio de Janeiro: Guanabara Koogan, pp. 241-268.

10 Sackman J.E. 1997. Pain and its management. Veterinary Clinics of North America, Small Animal practice. 27: 14871504.

11 Valadão A.A.C. 2002. Anestésicos dissociativos. In: Fantoni T.D. \& Cortopassi G.R.S. (Eds). Anestesia em cães e gatos. São Paulo: Roca, pp.165-173 